1 Universidade Federal de Santa Maria (UFSM) Santa Maria (RS), Brasil. dionatandelevati@gmail.com

\section{Desafios na gestão de resíduos de estabelecimentos de saúde públicos perante a RDC 222/18}

\author{
Waste management challenges of public health facilities subject to \\ RDC 222/18 \\ Dionatan dos Santos Delevati', Maria Manuela Ritondale Sodre de Castro' ${ }^{\mathbf{1}}$, Edi Franciele Ries ${ }^{\mathbf{1}}$, \\ Valéria Maria Limberger Bayer', Verginia Margareth Possatti Rocha'
}

DOI: $10.1590 / 0103-110420195314$

RESUMO A RDC 222/18 representa um avanço na área da gestão dos resíduos de serviços de saúde, no entanto, alguns desafios precisam ser considerados para o alcance dos impactos esperados na saúde ambiental e na qualidade de vida da população. Nesta perspectiva, considerando que estabelecimentos de saúde públicos precisam ser regulados pelo órgão responsável no que se refere às adequações sanitárias, o presente estudo tem como propósito relatar a experiência acadêmica de um profissional de saúde na coleta de dados de inspeção sanitária para pesquisa nos estabelecimentos de saúde públicos de um município do Sul do Brasil e os desafios ante a RDC 222/18. Como material de observação, foi utilizado o roteiro de inspeção aplicado em 28 estabelecimentos de saúde públicos. As informações coletadas foram categorizadas conforme os desafios observados. Verificaram-se desafios na gestão dos Resíduos de Serviços de Saúde (RSS) relativos à ausência de plano de gerenciamento, falha no manejo, falta de abrigo externo, falhas na tomada de decisão e quanto à responsabilidade técnica. Evidenciou-se que, para o cumprimento da RDC 222/18 e seus consequentes impactos positivos, o tema necessita de maior visibilidade em meio aos profissionais da saúde, especialmente no campo da educação permanente.

PALAVRAS-CHAVE Resíduos de Serviços de Saúde. Vigilância sanitária. Inspeção sanitária. Saúde ambiental.

ABSTRACT RDC 222/18 represents an advancement in the area of health care waste management, however, some challenges need to be considered in order to achieve the expected impact on environmental health and quality of life of the population. In this perspective, considering that public health institutions need to be regulated by the body responsible for sanitary management, this study aims to report the academic experience of a health professional in gathering data related to sanitary inspections of public health services in a municipality in Southern Brazil and the challenges facing RDC 222/18. As an observation material, the inspection list applied in 28 public health care facilities were used. The obtained information was categorized according to the observed challenges. There were challenges in the management of Health Service Waste (RSS) related to the absence of a management plan, management failure, lack of external shelter, decision-making failures and technical responsibility. It was noted that, in order to comply with RDC 222/18 and its consequent positive impacts, this theme needs greater visibility amongst health professionals, particularly in the permanent education field.

KEYWORDS Medical waste. Health surveillance. Sanitary inspection. Environmental health. 


\section{Introdução}

Os Resíduos de Serviços de Saúde (RSS), quando gerenciados inadequadamente, em quaisquer de seus processos de manejo, podem causar diversos danos à natureza, bem como ao meio ambiente, poluindo água, solo e ar, alterando fatores químicos, físicos e microbiológicos ambientais. Mesmo representando 1\% de todos os resíduos gerados no País, os RSS são particularmente importantes pelo risco potencial que apresentam à saúde pública e ao meio ambiente'.

$\mathrm{O}$ artigo $\mathrm{n}^{0} 13$ da Lei Federal $\mathrm{n}^{\circ}$ 12.305/2010, que trata da Política Nacional de Resíduos Sólidos (PNRS), define RSS² como aqueles que são gerados nas instituições que prestam serviços de saúde, conforme definido em regulamento ou em normas estabelecidas pelos órgãos do Sistema Nacional de Meio Ambiente (Sisnama) e do Sistema Nacional de Vigilância Sanitária (SNVS).

Os RSS são potenciais fontes de riscos para a saúde de quem os manuseia, tanto no ambiente interno quanto externo dos estabelecimentos geradores ${ }^{3}$. Adicionalmente, devido às suas características físicas, químicas e biológicas, bem como aos riscos envolvidos, faz-se indispensável preconizar nos Serviços de Saúde (SS) público e privado a sua adequada segregação pelos profissionais de saúde e funcionários, evitando, assim, os riscos ao meio ambiente e à saúde pública ${ }^{4}$.

Destaca-se que o serviço gerador de resíduos é responsável por elaboração, implantação, implementação e monitoramento do Plano de Gerenciamento de Resíduos dos Serviços de Saúde (PGRSS), sendo possível a terceirização de sua elaboração, implantação e monitoramento ${ }^{5}$.

O PGRSS é um pré-requisito documental para dar andamento ao processo de licenciamento sanitário, para obtenção do alvará sanitário municipal em estabelecimentos do setor privado, entretanto, os estabelecimentos de saúde públicos, mesmo não necessitando do alvará sanitário, precisam ser regulados pelo órgão responsável quanto às adequações sanitárias. Entre os parâmetros sanitários nele avaliados, encontra-se a gestão de RSS 5 .

Assim, conforme pactuação firmada entre estado e município, surge a Resolução da Comissão Intergestores Bipartite $\mathrm{n}^{0} 30$, de 11 de março de $2004^{6}$ (RS), definindo que a fiscalização dos estabelecimentos de saúde públicos fica a cargo da vigilância em saúde municipal.

A Resolução da Diretoria Colegiada (RDC) $\mathrm{n}^{\circ} 222$, de 28 de março de 2018, da Anvisa, regulamenta as boas práticas de gerenciamento dos RSS, dá outras providências ${ }^{5}$ e revoga a RDC n ${ }^{\circ} 306$, de 7 de dezembro de 2004, da Anvisa, que dispunha sobre o Regulamento Técnico para o gerenciamento de RSS ${ }^{7}$. Para orientação do tratamento e disposição final, permanece a Resolução do Conselho Nacional do Meio Ambiente $\mathrm{n}^{0}$ 358, de 29 de abril de 2005 (Conama 358/05) ${ }^{8}$, como instrumento de orientação, a fim de minimizar e/ou eliminar danos à saúde dos trabalhadores e ao meio ambiente.

Conforme destacado por Zanatta et al. ${ }^{9}$, a $\mathrm{RDC} 222 / 18$ representa avanço na busca da regulamentação e das boas práticas no tratamento de RSS, em direção ao desenvolvimento sustentável. Contudo, as dificuldades na fiscalização, no cumprimento da legislação e em possíveis punições aos estabelecimentos que não se ajustarem à $\mathrm{RDC}$ vigente perduram como desafios importantes para os órgãos fiscalizadores.

Considera-se que a colaboração dos profissionais envolvidos na gestão de RSS, adequações frente à legislação vigente e o conhecimento dos desafios dos gestores da área da saúde e órgãos fiscalizadores são essenciais para atingir os resultados positivos, tanto na saúde pública quanto no meio ambiente, esperados pela RDC 222/18. Assim, o presente estudo tem como propósito relatar a experiência acadêmica de um profissional de saúde na coleta de dados de inspeção sanitária para pesquisa nos estabelecimentos de saúde públicos de um município do Sul do Brasil, de acordo com as premissas preconizadas na RDC 222/18, apresentando, quando pertinente, paralelos com a RDC 306/04. 


\section{Métodos}

O relato refere-se à descrição da experiência acadêmica de um profissional de saúde na coleta de dados de inspeção sanitária para uma pesquisa descritiva exploratória, com observação participante, em serviços de saúde públicos de um município do Sul do Brasil.

O estudo foi realizado no município de Santa Maria, localizado no Centro do estado do Rio Grande do Sul, com uma população estimada de 280.505 habitantes $^{10}$ e que é considerado área de referência em assistência à saúde ${ }^{11}$. Segundo a Secretaria Municipal de Saúde de Santa Maria (SMSSM), existem 24 Unidades Básicas de Saúde (UBS), 8 Unidades Distritais (UD), 14 Unidades de Estratégia Saúde da Família (ESF) e 1 Centro de Especialidades Odontológicas (CEO) ${ }^{\mathbf{1 2}}$.

A RDC 222/18 não diferencia serviços geradores de RSS quanto à esfera administrativa ou natureza da organização, devendo ser igualmente aplicada a todos os serviços que geram resíduos ${ }^{5}$. Considerando estabelecimento de saúde o "espaço físico delimitado e permanente onde são realizadas ações e serviços de saúde humana sob responsabilidade técnica"13(2), foram incluídos no estudo 28 estabelecimentos de saúde do município com elevado volume de resíduos, selecionados de acordo com os critérios: i. realizar atendimento ao paciente; e ii. servir de espaço para ensino, pesquisa e extensão em diferentes cursos da área da saúde. Foram excluídos do estudo os estabelecimentos de saúde mental, de testagem e aconselhamento e de caráter excepcionalmente administrativo.

As visitas aos estabelecimentos de saúde foram realizadas durante o segundo semestre do ano de 2018, sem agendamento prévio, seguindo metodologia do dia típico, a fim de não estabelecer um possível efeito de expectativa. A logística das inspeções foi definida por conveniência pelo responsável pelo transporte, visando a otimizar tempo e trajeto, conforme os critérios existentes do transporte, de forma que a rotina da fiscalização não fosse alterada.
No local, realizou-se a identificação e solicitou-se a presença do enfermeiro responsável pelo estabelecimento. Em seis estabelecimentos, não havia presença desse profissional, e a inspeção foi acompanhada ora por técnico de enfermagem, ora por atendente administrativo.

A inspeção seguiu um roteiro adaptado e pré-estruturado pelas vigilâncias municipal e estadual, somado ao registro fotográfico, a fim de estabelecer um diagnóstico situacional dos estabelecimentos de saúde públicos quanto aos RSS. Os dados foram coletados e registrados pelo mesmo profissional responsável pela fiscalização em todos os estabelecimentos, para evitar viés do entrevistador.

Como instrumento de diagnóstico, foi utilizado 'roteiro de autoinspeção sanitária', desenvolvido no ano de 2018 com base nas legislações sanitárias vigentes, com o objetivo de nortear as inspeções sanitárias nos estabelecimentos de saúde públicos, considerando a variedade de serviços oferecidos nesses locais. Entre os itens do roteiro, encontram-se questões sobre existência do PGRSS, responsabilidade da gestão dos resíduos, resíduos odontológicos, transporte interno e externo, entre outros.

O fluxo da inspeção sanitária para a coleta de dados manteve direção única, iniciando com manejo, acondicionamento, armazenamento temporário e externo, quando houvesse, bem como a verificação contratual com empresa habilitada para a coleta interna, transporte externo, tratamento e disposição final, verificando-se possíveis falhas no processo de gestão dos RSS, apontando potencialidades e adequações.

A pesquisa foi autorizada pelo Núcleo de Educação Permanente em Saúde de Santa Maria (NEPeS) e aprovada em seus aspectos éticos pelo Comitê de Ética em Pesquisa da Universidade Federal de Santa Maria (CAAE: 97453318.9.0000.5346/Parecer $\mathrm{n}^{\circ}$ 2.958.973/2018).

Como técnica complementar ao roteiro de inspeção, foi utilizada a observação participante ${ }^{\mathbf{1 4}}$, com finalidade de obter informações das condições sanitárias e do gerenciamento dos resíduos relacionadas com a atuação dos 
membros da equipe de saúde ante a RDC $222 / 18$. As informações foram avaliadas através da análise de conteúdo do tipo temática ${ }^{15}$ para a estruturação das categorias nas quais identificaram-se desafios na inspeção perante a RDC 222/18.

Os dados coletados para levantamento do diagnóstico situacional dos estabelecimentos de saúde inspecionados e a análise de conteúdo das observações do profissional subsidiaram a descrição do relato de experiência.

Ao finalizar a exploração do material, os elementos constitutivos das observações foram agrupados por analogia e nortearam a categorização dos desafios identificados na inspeção sanitária: i. implantação e monitoramento do PGRSS; ii. tomada de decisão; iii. manejo dos RSS; iv. coleta e transporte interno dos RSS; v. infraestrutura dos estabelecimentos de saúde; e vi. responsabilidade técnica.

\section{Resultados e discussão}

\section{Desafios na implantação e no moni- toramento do PGRSS}

Durante o diagnóstico, foi possível verificar que falta sensibilização e informação, bem como há pouca integração nas equipes dos estabelecimentos de saúde públicos incluídos no estudo. Observou-se que, em 15 unidades $(53,57 \%)$, as quais possuem serviço de radiologia odontológica, o profissional enfermeiro parecia desconhecer a gestão de RSS relacionados ao setor odontológico. Considerando que resíduos odontológicos também são RSS, toda a equipe deve ser responsável pelo seu gerenciamento, assim como dos demais resíduos ${ }^{5}$.

Em estudo realizado no município de São Paulo, Brasil, foi verificado que determinantes essenciais para alavancar a implantação do PGRSS nos estabelecimentos de saúde públicos foram: vontade política e tomada de decisão, recursos humanos e econômicos, informação, capacitação e maior integração entre os profissionais envolvidos ${ }^{16}$. Segundo Zanatta et al. ${ }^{9}$, o PGRSS é uma ferramenta importante que contribui para diminuir a quantidade de resíduos a serem tratados. A existência e o cumprimento desse plano permitem, por meio do planejamento, da avaliação constante e da adequação, que o gestor acompanhe o processo através de indicadores, realizando constantes melhorias.

O PGRSS deve ser monitorado e atualizado conforme periodicidade definida pelo responsável pela elaboração e pela implantação $0^{5}$. A RDC 306/04 orientava o gerador ao desenvolvimento de instrumentos de avaliação e controle, incluindo construção de indicadores claros, autoexplicativos e confiáveis, permitindo acompanhar a eficácia do PGRSS implantado. Porém, na RDC 222/18 não são descritos indicadores a serem trabalhados. Apenas no artigo $\mathrm{n}^{\circ} 19$ é apontado 'conhecimento dos instrumentos de avaliação e controle do PGRSS', tema a ser desenvolvido em atividades de educação permanente.

\section{Desafios na tomada de decisão}

Das 28 unidades inspecionadas, apenas duas (7,14\%) apresentaram o PGRSS na inspeção. Parte dos profissionais questionados sobre o PGRSS referiu não saber do que se tratava; outra parte comentou não ser contemplada com capacitação permanente sobre os RSS. Verificou-se que o desconhecimento da legislação ou a inobservância e a dificuldade do acesso podem ser fatores que dificultam a tomada de decisão das equipes de saúde na gestão de resíduos.

Desta forma, observa-se uma integração e corresponsabilização necessárias entre os profissionais envolvidos, no que tange à tomada de decisão no processo de trabalho dos estabelecimentos inspecionados. Por esse prisma, a adequada gestão dos RSS parte, inicialmente, da necessidade de se desenvolver um PGRSS adequado, formatado e pactuado entre os profissionais envolvidos, à luz da legislação vigente.

Os serviços de saúde devem trabalhar de modo interdisciplinar, cooperando na gestão e nas decisões. Assim, caminha-se para 
reorganização da atenção primária à saúde cultivando trabalho em equipe multiprofissional e interdisciplinar para desenvolver práticas gerenciais e assistenciais, em que todos os atores são responsáveis pela saúde da população ${ }^{17}$.

Considerando a abrangência que trata a nova legislação, é importante a clareza em suas normatizações para compreensão de todos. A RDC 306/04 apresentava orientações que eram retomadas no transcorrer de redação, tornando-se evidente que, mesmo parecendo repetitiva, foi elaborada para contribuir para o esclarecimento das informações vinculadas ${ }^{7}$. Em contrapartida, a RDC 222/18 apresenta conceitos prévios apenas no início de seu conteúdo, não recapitulando os itens no decorrer da leitura ${ }^{5}$. Assim, compreende-se que, na produção textual, a repetição de itens faz parte de uma construção argumentativa que permite que o significado das palavras se transforme conforme a redação vai se desdobrando, produzindo novas formas de interpretação do que foi dito no material, sendo assim, é importante a existência de elementos de recorrência para, dessa forma, resultar em um texto mais coerente ${ }^{\mathbf{1 8}}$.

\section{Desafios no manejo dos RSS}

Dos estabelecimentos de saúde incluídos no estudo, 13 (46,42\%) apresentaram falhas na segregação dos RSS, favorecendo exposição a riscos ambientais e aumento de custos associados. Entre as inadequações nessas unidades, foi observada a presença de resíduos contaminados em coletores de resíduos comuns, o que pode ser consequência da não identificação dos coletores, levando o profissional de saúde à confusão no momento da segregação.

$O$ fato de a RDC 222/18 dispensar a identificação dos sacos do resíduo do grupo $\mathrm{D}$, sem descrever cor adequada para acondicionamento ${ }^{5}$, é um desafio no manejo dos resíduos. Já a RDC 306 obrigava identificação e sacolas específicas ${ }^{7}$. Portanto, a sensibilização das pessoas envolvidas no manejo dos resíduos é essencial para sua diminuição, corroborando apontamentos tratados na RDC 222/18, de que as formas de reduzir a geração de RSS devem ser tratadas no programa obrigatório de educação continuada 5 .

Outrossim, ocorre um contraponto com relação à RDC 222/18 e NBR 9.191 ${ }^{19}$, que determina que 'sacos plásticos classe I podem apresentar qualquer cor, exceto branca; sacos classe II só podem apresentar a cor branca leitosa', prejudicando a tomada de decisão e a segregação adequada de resíduos ${ }^{5,20}$.

Durante o levantamento, foi observado que, nos estabelecimentos que não contam com abrigo externo, é utilizada área externa para disposição dos coletores, muitas vezes colocando em risco a população e o meio ambiente ${ }^{6}$. $\mathrm{Na}$ RDC 222/18, o armazenamento temporário pode não necessariamente ocorrer em abrigo externo ${ }^{5}$. Dependendo do estabelecimento de saúde inspecionado, o acondicionamento dos resíduos ocorre em área de lavagem, que se caracterizaria em uma sala de utilidades, no entanto, muitas vezes, esse espaço é limitado, não sendo possível 0 acondicionamento de bombonas.

Foi observado no estudo que nenhum dos estabelecimentos de saúde realizava tratamento prévio dos resíduos infectantes, deixando a cargo da empresa contratada para a coleta interna o transporte externo, o tratamento e a disposição final. A RDC 222/18 recomenda que resíduos do grupo A, quando forem encaminhados para incineração, devem ser acondicionados em sacos da cor vermelha, caso contrário, podem ser segregados no saco branco leitoso, quando houver normativas locais obrigando o tratamento indiscriminado de todos os resíduos do grupo 20 .

\section{Desafios na coleta e transporte inter- no dos RSS}

Apenas um dos estabelecimentos de saúde inspecionados no estudo possuía carro de coleta de RSS. O transporte era realizado manualmente pelos profissionais da limpeza, sem controle de horário e rota de coleta da empresa contratada, tendo acesso por dentro da unidade ou externamente. 
A coleta e o transporte interno dos RSS são o trajeto destes da geração até o armazenamento temporário ou externo, permanecendo disponíveis para coleta externa. É nessa fase que o processo se torna visível para o público em geral, pois o transporte é realizado em carros de coleta em áreas comuns do serviço ${ }^{20}$.

Segundo Vilela e Angra Filho ${ }^{21}$, é essencial que no PGRSS conste se a coleta e o transporte interno são realizados manual ou mecanicamente, descrevendo os tipos de instrumentos, Equipamento de Proteção Individual (EPI) e recipientes utilizados, além da rota a ser realizada, partindo do acondicionamento até o local do armazenamento temporário, prevendo horários, frequência, não coincidindo com os horários de maior fluxo de pessoas na unidade. Essas informações são muito importantes para o transporte seguro dos resíduos até o armazenamento externo.

Em oito estabelecimentos de saúde (28,57\%) inspecionados, os profissionais referiram dificuldades quanto à frequência de coleta da empresa contratada, geralmente ocasionando extrapolamento de carga no abrigo externo, impossibilitando até mesmo o seu fechamento. O município conta com dois fiscais de contrato, este referente à empresa prestadora de serviço contratada para a coleta, que devem ser contatados em caso de dúvidas e informados quando há suspeita de irregularidade, podendo minimizar as dificuldades relacionadas à frequência de coleta.

\section{Desafios de infraestrutura dos esta- belecimentos de saúde}

Durante as inspeções, verificou-se que 12 unidades $(42,85 \%)$ apresentavam abrigo externo. Porém, nenhuma apresentou característica compatível com a legislação atual. Observou-se a existência de pisos, paredes e ralos inadequados e ausência de identificações nas aberturas. Os abrigos, em geral, mantiveram o uso de apenas um dos lados do abrigo, deixando o outro, destinado a resíduos comuns, ocupado com materiais inservíveis advindos da unidade.
A instalação do abrigo externo é obrigatória em estabelecimentos de saúde públicos, conforme Portaria Federal $n^{\circ} 340$, de 04 de março de 2013, sendo descritas na RDC 306/04 duas possibilidades, conforme o volume de resíduos gerados e a periodicidade de coleta: i. abrigo externo; ou ii. abrigo reduzido exclusivo, apresentando menor tamanho7. A legislação em vigor não prevê abrigo reduzido exclusivo ${ }^{5}$.

Um estudo ${ }^{22}$ realizado em 34 municípios da região metropolitana de Belo Horizonte/ MG avaliou 54 estabelecimentos de saúde e levantou a possível inobservância por parte dos municípios com relação ao atendimento aos requisitos da legislação pertinente, visto que poucas unidades se mostraram adequadas quanto ao abrigo externo, pois muitas nem o possuíam, mantendo os RSS expostos ao tempo, a curiosos e até a catadores, que desconhecem os riscos inerentes a esse tipo de resíduo. Outro estudo ${ }^{23}$, realizado em 18 UBS na cidade de Pelotas (RS), evidenciou que as condições de armazenamento temporário interno e externo nem sempre estão de acordo com o que recomenda a legislação vigente, podendo oferecer riscos ao ambiente e à população exposta.

A pesquisa de Ramos e colaboradores ${ }^{24}$, realizada em serviços de saúde de João Pessoa (PB), evidenciou que $100 \%$ dos depósitos de RSS mostravam-se inadequados, apresentando deficiências em suas instalações elétricas e hidráulicas, ausência de ventilação e baias não individualizadas para separar os vários tipos de resíduos (comuns e infectantes), descumprindo as normativas legais vigentes, colocando em risco o meio ambiente e a saúde dos trabalhadores.

Entretanto, a RDC 222/18 não obriga que o abrigo externo seja de alvenaria. Dessa forma, dificulta-se a compreensão de como devem ser as instalações do abrigo externo, comprometendo o entendimento de uma situação que já era precária 5 .

Durante a inspeção realizada no presente estudo, verificou-se que a quantidade gerada de resíduos do grupo E (perfurocortante) nos estabelecimentos de saúde resulta na 
impossibilidade do acondicionamento das caixas de perfurocortante dentro dos coletores do abrigo externo, mantendo, algumas vezes, o armazenamento dessas caixas diretamente no chão do abrigo, o que pode comprometer o material segregado. Além de não estar de acordo com a legislação vigente ${ }^{5}$, tal condição pode provocar acidentes e ou contaminação dos profissionais de saúde, dos funcionários da limpeza, bem como dos coletores de resíduos ${ }^{4}$.

Levando-se em consideração que certos estabelecimentos de saúde públicos separam os frascos íntegros dos demais resíduos do grupo E, com intuito de reciclagem, isso pode potencializar o acúmulo de caixas coletoras, visto que o município não conta com contrato com empresa de reciclagem, contrato este que deveria estar contemplado no PGRSS, conforme RDC 222/18 .

\section{Desafios da responsabilidade técnica}

Não está prevista em lei a indicação de um responsável técnico para os estabelecimentos de saúde públicos, entretanto, verifica-se, na prática, essa necessidade. Assim, em geral, ocorre um distanciamento entre os profissionais de nível superior quando se deseja implementar um serviço que envolva a participação de todos os atores, como no caso do processo de criação do PGRSS. Neste sentido, percebe-se a dificuldade de apontar os responsáveis quando existe um descumprimento da norma sanitária, não sendo possível, muitas vezes, estabelecer punições, como se observa na iniciativa privada.

Conforme Zanatta et al. ${ }^{9}$, a lei que estabelece a PNRS não é muito específica quanto às punições ou restrições estabelecidas em caso de descumprimento da norma. No entanto, o rito do processo administrativo sanitário é o mesmo para qualquer descumprimento legal estabelecido no âmbito nacional pela Lei Federal $n^{\circ} 6.437 / 97^{25}$, que configura as infrações à legislação sanitária para aqueles municípios que não possuam sua própria legislação prevendo as penalidades. No caso do município de Santa Maria, as normas de saúde em vigilância sanitária e suas penalidades são previstas pela lei Municipal no 4.040/9626.

Para o gerador de RSS, é indispensável o contrato com empresas legalizadas que prestam serviços de coleta e destinação dos resíduos. A Lei no ${ }^{0}$ 12.305/2010 da PNRS é clara quando diz que o gerador é responsável pelo resíduo da geração até a disposição final².

Desta forma, para exercer a função de responsável pela elaboração e pela implantação do PGRSS, na RDC 306/04, o profissional deveria apresentar registro ativo junto ao seu conselho de classe, com apresentação de anotação de responsabilidade técnica-ART, ou certificado de responsabilidade técnica ou documento similar, quando coubesse ${ }^{8}$. No entanto, a RDC 222/18, no art. 10, preconiza que o serviço gerador de RSS é responsável por elaboração, implantação, implementação e monitoramento do PGRSS, não definindo critérios de responsabilidade individuais, e, sim, a corresponsabilização. Assim, a responsabilidade compartilhada estende-se a outros atores: poder público e empresas de coleta, tratamento e disposição final ${ }^{5}$.

\section{Considerações finais}

Percebe-se que são vários os desafios que esses estabelecimentos estão enfrentando quanto à utilização da legislação em vigor como base para seu processo de trabalho, considerando a gestão dos RSS, comprometendo, assim, a implantação do PGRSS.

Acredita-se que esse cenário possivelmente está relacionado com a falta de capacitação sobre o gerenciamento dos RSS, somada à baixa relevância dada ao assunto e ao descumprimento do recomendado em legislação.

Diante das fragilidades encontradas na gestão dos RSS, propõe-se não apenas investimento público na gestão de resíduos, mas, também, uma maior aproximação dos profissionais dos SS com a vigilância sanitária municipal, através da educação sanitária, 
melhoria na infraestrutura, bem como maior controle no gerenciamento dos resíduos, da coleta externa até o destino final, minimizando os riscos à saúde pública e ao meio ambiente.

O estudo apresenta como limitação a restrição quanto à pesquisa do tipo relato de experiência, e, como sugestão para estudos futuros, tem-se a gestão de resíduos provenientes de atividades odontológicas e a avaliação do impacto da educação permanente em saúde na melhoria da gestão de resíduos em estabelecimentos de saúde.

Apesar de algumas dificuldades quanto ao entendimento da RDC 222/18, que refletem em desafios na tomada de decisão na equipe, ela representa um avanço importante na gestão dos RSS no que tange à diminuição e ao encaminhamento seguro e eficiente de resíduos, impactando na saúde ambiental e coletiva, bem como em maior qualidade de vida da população.
A efetivação deste estudo foi viabilizada pela parceria entre universidade e prefeitura, possibilitando o compartilhamento da construção do conhecimento e de experiências sobre a temática, cenário este impossibilitado quando instituições de ensino e serviços públicos atuam de forma independente.

\section{Colaboradores}

Delevati DS (0000-0003-2445-5555)*, Castro MMRS (0000-0002-7775-5765)*, Ries EF (0000-0001-6688-2913)*, Bayer VML (00000002-6731-4835)* e Rocha VMP (0000-00028062-095X)* contribuíram igualmente para concepção, planejamento, análise e interpretação dos dados; elaboração do rascunho, revisão crítica do conteúdo e aprovação da versão final do manuscrito. 


\section{Referências}

1. Costa VM, Batista NJC. Gerenciamento de resíduos de serviço de saúde: uma revisão integrativa. Rev. Saúde em Foco. [internet]. 2016 [acesso 2019 ago 21]; 3(1):124-145. Disponível em: http://www4.fsanet.com. br/revista/index.php/saudeemfoco/article/download/952/1006.

2. Brasil. Lei ${ }^{0} 12.305$, de 2 de agosto de 2010. Institui a Política Nacional de Resíduos Sólidos; altera a Lei $\mathrm{n}^{\circ}$ 9.605, de 12 de fevereiro de 1998; e dá outras providências. Diário Oficial da União. 3 Ago 2010.

3. Gallotti FCM, Santos ATS, Oliveira CB, et al. Gerenciamento de resíduos de serviços de saúde: conhecimento da equipe de enfermagem. Ciências Biológicas e de Saúde Unit [internet]. 2017 [acesso 2019 ago 21]; 4(2):169-164. Disponível em: https://periodicos.set.edu.br/index.php/cadernobiologicas/article/ view $/ 4600 / 2502$.

4. Uehara SCSA, Veiga TB, Takayanagui AMM. Gerenciamento de resíduos de serviços de saúde em hospitais de Ribeirão Preto (SP), Brasil. Eng. Sanit. Ambient. [internet]. 2019 [acesso 2019 ago 21]; 10(10):121-130. Disponível em: http://dx.doi.org/10.1590/s141341522019175893.

5. Agência Nacional de Vigilância Sanitária. Resolução da Diretoria Colegiada n 222, de 28 de março de 2018. Regulamenta as Boas Práticas de Gerenciamento dos Resíduos de Serviços de Saúde e dá outras providências. Diário Oficial da União. 29 Mar 2018.

6. Rio Grande do Sul. Secretaria de Saúde. Resolução da Comissão Intergestores Bipartite $n^{\circ} 30$, de 11 de março de 2004. Dispõe sobre os estabelecimentos de baixa complexidade. Diário Oficial do Estado. 12 Mar 2004.

7. Agência Nacional de Vigilância Sanitária. Resolução da Diretoria Colegiada n ${ }^{\circ}$ 306, de 7 de dezembro de 2004. Dispõe sobre o Regulamento Técnico para o gerenciamento de resíduos de serviços de saúde. Diário Oficial da União, 22 Dez 2000.
8. Brasil. Ministério do Meio Ambiente. Resolução do Conselho Nacional do Meio Ambiente n ${ }^{\circ}$ 358, de 29 de abril de 2005. Dispõe sobre o tratamento e a disposição final dos resíduos de saúde e dá outras providências. Diário Oficial da União. 29 Abr 2005.

9. Zanatta JM, Halberstadt IA, Dias DO, et al. Análise crítica da RDC-222/2018 à luz das dimensões do desenvolvimento sustentável: avanços e desafios. Rev. da Universidade Vale do Rio Verde [internet]. 2019 [acesso 2019 mar 20]; 17(1):1-17. Disponível em: http:// periodicos.unincor.br/index.php/revistaunincor/article/view/4967/pdf_911.

10. Instituto Brasileiro de Geografia e Estatística. Estatísticas [internet]. Cidades e Estados [acesso em 2019 abr 25]. Disponível: https://www.ibge.gov.br/ cidades-e-estados/rs/santa-maria.html?.

11. Rio Grande do Sul. Secretaria Municipal de Saúde de Santa Maria. Plano Municipal de Saúde [internet]. Plano Municipal de Saúde. [acesso em 2019 ago 21]. Disponível: https://www.santamaria.rs.gov.br/ saude/650-plano-municipal-de-saude-20092012.

12. Rio Grande do Sul. Secretaria Municipal de Saúde de Santa Maria. Postos de Saúde [internet]. Postos de Saúde. [acesso em 2019 abr 25]. Disponível: https://www.santamaria.rs.gov.br/saude/191-postos-de-saude.

13. Brasil. Ministério da Saúde. Portaria $n^{0} 1.646$, de 2 de outubro de 2015. Institui o Cadastro Nacional de Estabelecimentos de Saúde (CNES), Diário Oficial da União. 5 Out 2015.

14. Minayo MCS. O desafio do conhecimento: pesquisa qualitativa em saúde. São Paulo; Rio de Janeiro: Hucitec; Abrasco; 1992.

15. Bardin L. Análise de conteúdo. 4. ed. Lisboa: Edições $70 ; 2010$.

16. Moreira AMM, Günther WMR. Gerenciamento de 
resíduos de serviços de saúde: aplicação de um instrumento facilitador. Rev. Latino-Am. Enfermagem [internet]. 2016 [acesso em 2019 ago 21]; 24(e2768):19. Disponível em: http://dx.doi.org/10.1590/15188345.0646.2768.

17. Penedo RM, Gonçalo CS, Queluz DP. Gestão compartilhada: percepções de profissionais no contexto de Saúde da Família. Interface [internet]. 2018 [acesso em 2019 ago 21]; 23(e170451):1-15. Disponível em: http://dx.doi.org/10.1590/interface.170451.

18. Antunes I. A coesão como propriedade textual: bases para o ensino do texto. Calidoscópio [internet]. 2009 [acesso em 2019 ago 21]; 7(1):1-10. Disponível em: http://revistas.unisinos.br/index.php/calidoscopio/article/view/4855/2113.

19. Associação Brasileira de Normas Técnicas. NBR 9.191: sacos plásticos para acondicionamento de lixo - Requisitos e métodos de ensaio. Rio de Janeiro: ABNT; 2008.

20. Agência Nacional de Vigilância Sanitária. Resolução da Diretoria Colegiada no 222/2018 Comentada, de 11 de junho de 2018 [internet]. RDC 222 comentada. 282018 [acesso em 2019 ago 21]. Disponível em: https://www20.anvisa.gov.br/segurancadopaciente/ index.php/legislacao/item/resolucao-rdc-n-222-de-28-de-marco-de-2018-comentada.

21. Vilela DMOC, Agra Filho SS. Análise de planos de gerenciamento de resíduos de serviços de saúde em hospitais em Salvador, Bahia. Rev. Eletrônica de Gestão e Tecnologias Ambientais [internet]. 2019 [acesso em 2019 mar 27]; 7(1):61-75. Disponível em: https://portalseer.ufba.br/index.php/gesta/article/ view/28072/17953.
22. Silva DF, Sperling EV, Barros RTV. Avaliação do gerenciamento dos resíduos de serviços de saúde em municípios da região metropolitana de Belo Horizonte (Brasil). Eng. Sanit. Ambient. [internet]. 2014 [acesso em 2019 ago 21]; 19(3):251-262. Disponível em: http://www.scielo.br/pdf/esa/v19n3/1413-4152esa-19-03-00251.pdf.

23. Neves BC, Lima EPP. Condições da prestação dos serviços ambientais de coleta e destinação de resíduos de serviços de saúde em unidades básicas de saúde na cidade de Pelotas, RS, Brasil. Eng. Sanit. Ambient. [internet]. 2019 [acesso em 2019 ago 21]; 24(1):6169. Disponível em: http://www.scielo.br/pdf/esa/ v24n1/1809-4457-esa-s1413-41522019172729.pdf.

24. Ramos YS, Pessoa YSRQ, Ramos YS, et al. Vulnerabilidade no manejo dos resíduos de serviços de saúde de João Pessoa (PB, Brasil). Ciênc. Saúde Colet. [internet]. 2011 [acesso em 2019 mar 27]; 16(8):35533560. Disponível em: http://www.scielo.br/pdf/csc/ v16n8/a23v16n8.pdf.

25. Brasil. Lei no 6437, de 20 de agosto de 1997. Configura infrações à legislação sanitária federal, estabelece as sanções respectivas, e dá outras providências. Diário Oficial da União. 20 Ago 1997.

26. Santa Maria. Lei no 4.040 de 27, de dezembro de 1996. Dispõe sobre normas de saúde em vigilância sanitária municipal, estabelece penalidades e dá outras providências. 27 Nov 1996. [acesso em 2019 mar 3]. Disponível em: https://camara-municipal-da-santa-maria. jusbrasil.com.br/legislacao/540470/lei-4040-96.

Recebido em 30/04/2019

Aprovado em 22/10/2019

Conflito de interesses: inexistente

Suporte financeiro: não houve 